\title{
Triple-deck and direct numerical simulation analyses of high-speed subsonic flows past a roughness element
}

\author{
G. Mengaldo ${ }^{1, \dagger}$, M. Kravtsova ${ }^{2}$, A. I. Ruban ${ }^{2}$ and S. J. Sherwin ${ }^{1}$ \\ ${ }^{1}$ Department of Aeronautics, Imperial College London, London SW7 2AZ, UK \\ ${ }^{2}$ Department of Mathematics, Imperial College London, London SW7 2AZ, UK \\ (Received 16 December 2014; revised 22 April 2015; accepted 13 May 2015; \\ first published online 9 June 2015)
}

This paper is concerned with the boundary-layer separation in subsonic and transonic flows caused by a two-dimensional isolated wall roughness. The process of the separation is analysed by means of two approaches: the direct numerical simulation (DNS) of the flow using the Navier-Stokes equations, and the numerical solution of the triple-deck equations. Since the triple-deck theory relies on the assumption that the Reynolds number $(R e)$ is large, we performed the Navier-Stokes calculations at $R e=4 \times 10^{5}$ based on the distance of the roughness element from the leading edge of the flat plate. This $R e$ is also relevant for aeronautical applications. Two sets of calculation were conducted with the free-stream Mach number $M a_{\infty}=0.5$ and $M a_{\infty}=0.87$. We used different roughness element heights, some of which were large enough to cause a well-developed separation region behind the roughness. We found that the two approaches generally compare well with one another in terms of wall shear stress, longitudinal pressure gradient and detachment/reattachment points of the separation bubbles (when present). The main differences were found in proximity to the centre of the roughness element, where the wall shear stress and longitudinal pressure gradient predicted by the triple-deck theory are noticeably different from those predicted by DNS. In addition, DNS predicts slightly longer separation regions.

Key words: boundary layer separation, compressible boundary layers, Navier-Stokes equations

\section{Introduction}

The triple-deck theory was specifically designed with the purpose of describing the phenomenon of boundary-layer separation at large values of the Reynolds number. The basic ideas that underlie the foundation of the triple-deck theory came from experimental studies of boundary-layer separation, including the separation caused by an impinging shock wave, with the then unexplained phenomenon of 'upstream influence'. These experiments are summarised by Chapman, Kuehn \& Larson (1958), and early theoretical models to explain the phenomenon are reviewed in a paper by Lighthill (2000). As a formal mathematical theory, the triple-deck theory was put forward by Neiland (1969), Stewartson (1969), Stewartson \& Williams (1969),

$\dagger$ Email address for correspondence: g.mengaldo11@imperial.ac.uk 
Messiter (1970) and Sychev (1972), and a detailed description of the basic aspects of the theory can be found in the monograph by Sychev et al. (1998). According to the triple-deck theory, the flow near the separation point is described by Prandtl's boundary-layer equations. The main alteration to Prandtl's classic theory is that the pressure acting on the boundary layer is not known in advance, but has to be found as part of the solution, as it is affected by the displacement effect of the boundary layer. The main advantage of the triple-deck theory is that there are no restrictions on how large the Reynolds number is. In fact, the larger the Reynolds number, the more accurate the theory is. However, numerical solution of the triple-deck equations is rather difficult, especially when the separation region is not small. It took a decade before reliable numerical techniques were developed; see chapter 7 in Sychev et al. (1998).

Numerical techniques for solving the Navier-Stokes equations have also undergone significant improvement over recent decades. A comprehensive summary of the role of direct numerical simulation (DNS) in fluid mechanics up to 1998 can be found in Moin \& Mahesh (1998) and references therein. More recently, compressible DNS has been performed in various works, among which we cite Pirozzoli, Grasso \& Gatski (2004), Martin (2007), Rizzetta et al. (2010) and Rizzetta \& Visbal (2014). Despite all of these advances, the task of calculating large-Reynolds-number separated flows, especially for transonic flow regimes, has been challenging. It is therefore not surprising that there are only a few publications where the Navier-Stokes calculations are compared with the triple-deck predictions; see, for example, Hsiao \& Pauley (1994).

In this paper, we use the subsonic and transonic versions of the triple-deck theory. The governing equations for the subsonic triple-deck theory were formulated by Neiland (1969), Stewartson (1969), Stewartson \& Williams (1969), Messiter (1970) and Sychev (1972). The transonic version of triple-deck theory was first formulated by Bodonyi \& Kluwick (1977), Bodonyi (1979) and Bodonyi \& Kluwick (1982, 1998). In our calculations we employed the special regime described by Timoshin (1990) and Bowles \& Smith (1993). Concerning the DNS, we solved the two-dimensional compressible Navier-Stokes equations by means of a high-order spectral/ $h p$ element approach, where we used a discontinuous Galerkin (DG) method in space and an explicit fourth-order Runge-Kutta scheme in time. The interested reader can refer to Karniadakis \& Sherwin (2005), Hesthaven \& Warburton (2008) and De Grazia et al. (2014).

The paper is organised as follows. In $\S 2$ we detail the model problem considered. In $\S 3$ we describe both the triple-deck model employed and the DNS tool used. In $\S 4$ we present the comparison results obtained on the model problem, and in $\S 5$ we briefly summarise the main conclusions of this work.

\section{Model problem}

The problem considered consists of a two-dimensional flat plate equipped with an isolated roughness element as shown in figure 1. We took into account various roughness heights as well as different free-stream conditions in order to investigate both linear and nonlinear regimes as well as subsonic (case 1) and transonic (case 2) conditions. The shape of the roughness element was given by

$$
F(x)=h_{r} \mathrm{e}^{-(L-x)^{2} / \beta^{2}},
$$

where $L$ is the distance from the centre of the roughness element to the leading edge of the flat plate, $h_{r}$ is the height of the roughness element and $\beta$ is a 


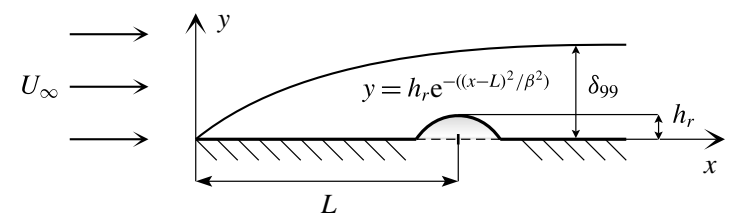

FIGURE 1. Model problem.

$\begin{array}{lccccc} & M a_{\infty} & T_{\text {wall }}(\mathrm{K}) & h_{r} & R e & P r \\ \text { Case 1 - Subsonic } & 0.50 & 216.29 & {[0.05,0.10,0.15] \delta_{99, L}} & 4 \times 10^{5} & 0.72 \\ \text { Case 2 - Transonic } & 0.87 & 258.0 & {[0.05,0.10,0.15] \delta_{99, L}} & 4 \times 10^{5} & 0.72\end{array}$

TABLE 1. Set of numerical simulations performed.

parameter that controls the shape of the roughness element. In particular, $L$ and $\beta$ were fixed and equal to $0.05 \mathrm{~m}$ and $1.7961 \times 10^{-4} \mathrm{~m}$, respectively. Concerning the height of the roughness element, we took into account three different values: $h_{r}=[0.05,0.10,0.15] \delta_{99, L}$, where $\delta_{99, L}$ is the thickness of the Blasius boundary layer at $x=L$. (Note that $\delta_{99, L}$ based on the Blasius solution is just a reference value. The corresponding values in terms of the compressible boundary-layer solutions are as follows: case $1, h_{r}=[0.042,0.084,0.126] \delta_{99, L, \text { comp }}$; case 2 , $\left.h_{r}=[0.039,0.078,0.118] \delta_{99, L, c o m p}.\right)$ The Reynolds number, defined as

$$
R e=\frac{\rho_{\infty} u_{\infty} L}{\mu_{\infty}},
$$

was set equal to $4 \times 10^{5}$ in all the tests performed, where, $\rho_{\infty}$ denotes the free-stream density, $u_{\infty}$ is the free-stream velocity and $\mu_{\infty}$ refers to the free-stream dynamic viscosity.

A summary of the main parameters used for the numerical simulations is reported in table 1. Note that we applied isothermal boundary conditions to the flat plate surface, where the wall temperature was equal to the far-field temperature in the subsonic case, while it was calculated using the Crocco integral in the transonic case.

The Mach, Reynolds and Prandtl numbers were chosen to be relevant for aeronautical applications, while the shape and the related parameters can be seen as simple models of small imperfections at the leading edge of civil aircraft wings. In addition, the three hump heights explored range from a non-separated flow to a well-established separation bubble behind the obstacle. Note that, in this paper, we are mainly interested in the nonlinear regime; therefore, the case $h_{r}=0.05 \delta_{99}$ was chosen such that it fell within a nonlinear regime without separation.

\section{Theoretical and numerical approaches}

\subsection{Triple-deck formulation}

The scales adopted in the triple-deck formulation are depicted in figure 2. Specifically, the subsonic case is represented in figure 2(a), while the transonic case is in figure $2(b)$. In the following, we briefly introduce the triple-deck formulation used in this paper. 

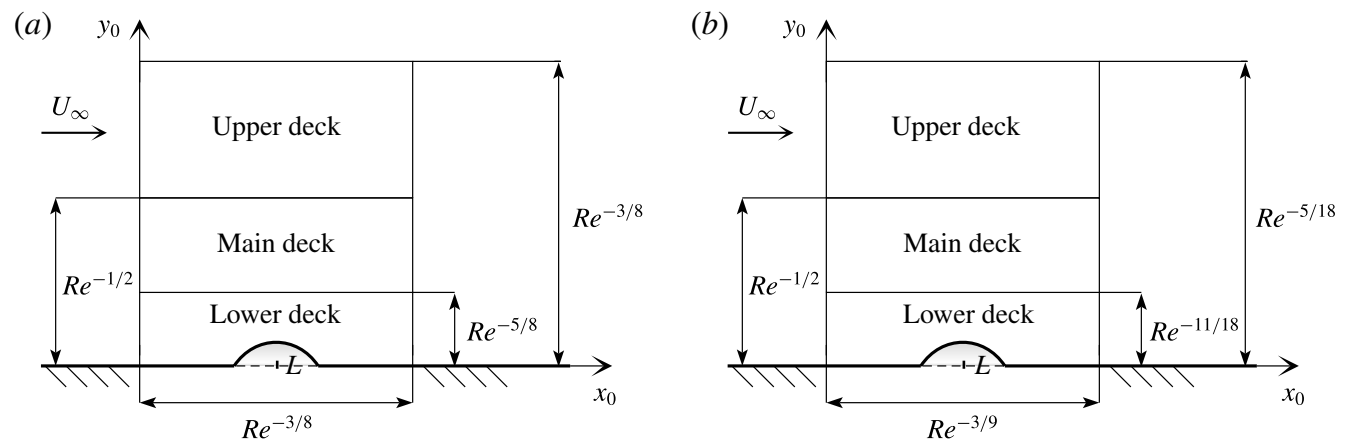

FIGURE 2. Three-tiered structure of the interaction region for $(a)$ the subsonic and $(b)$ the transonic cases. form

We first write the set of equations for the lower deck (see figure 2) in the canonical

$$
\left.\begin{array}{c}
u_{0} \frac{\partial u_{0}}{\partial x_{0}}+v_{0} \frac{\partial u_{0}}{\partial y_{0}}=-\frac{\partial p_{0}}{\partial x_{0}}+\frac{\partial^{2} u_{0}}{\partial y_{0}^{2}}, \\
\frac{\partial u_{0}}{\partial x_{0}}+\frac{\partial v_{0}}{\partial y_{0}}=0
\end{array}\right\}
$$

with the conditions

$$
\left.\begin{array}{ll}
u_{0}=v_{0}=0 & \text { at } y_{0}=0 \\
u_{0}=y_{0}+\cdots & \text { as } x_{0} \rightarrow-\infty \\
u_{0}=y_{0}+A_{0}(x)+\cdots & \text { as } y_{0} \rightarrow \infty
\end{array}\right\}
$$

where $A_{0}(x)$ is the displacement function, $x_{0}$ and $y_{0}$ are the streamwise and wallnormal coordinates, $u_{0}$ and $v_{0}$ are the associated velocities, $p_{0}$ is the pressure and the subscript ' 0 ' denotes leading-order dimensionless quantities, which are defined in the asymptotic expansions used and defined in $\$ \S 3.1 .1$ and 3.1.2. The interaction law between the boundary layer (lower deck) and the potential flow in the upper deck has the form of a Hilbert integral for subsonic flows. In the transonic case, the interaction law is instead represented by the following integral:

$$
p_{0}\left(x_{0}\right)=\frac{1}{\pi \sqrt{m}} \int_{-\infty}^{\infty} \frac{F^{\prime}(s)-A_{0}^{\prime}(s)}{s-x_{0}} \mathrm{~d} s .
$$

Here $F\left(x_{0}\right)$ is the shape of the roughness element defined in $\S 2$, and $m$ is known as the Kármán-Guderley parameter ( $m$ was equal to 1.3 in all the transonic cases taken into account) and is defined as

$$
M a_{\infty}^{2}=1-m R e^{-1 / 9}
$$

where $M a_{\infty}$ is the free-stream Mach number. Note that in (3.3), for the transonic case, the upper-deck steady problem is described by the transonic small perturbation (TSP) equation disregarding the time derivative (for additional details, the interested reader can refer to Cole \& Cook (1986)). In the subsonic case, the upper deck is instead solved through the Laplace equation for the pressure. 
To solve the steady triple-deck problem numerically, we discretise our twodimensional domain with a set of mesh points at generic coordinates $\left(x_{0, i}, y_{0, j}\right)$, where $\left(i=1, \ldots, N_{x_{0}} ; j=1, \ldots, N_{y_{0}}\right)$. Following Kravtsova, Zametaev \& Ruban (2005), where the numerical approach adopted here is detailed, we define the displacement function vector $\boldsymbol{A}_{0, i}$ and the pressure vector $\boldsymbol{p}_{0, i}$ at each point $x_{0, i}$. The vectors $\boldsymbol{A}_{0, i}$ and $\boldsymbol{p}_{0, i}$ define the values of the displacement function and of the pressure at all the points along the wall-normal direction $y_{0}$. For a given displacement function $\boldsymbol{A}_{0, i}$, the interaction law in (3.3) (equivalently the interaction law for the subsonic case) allows the calculation of the inviscid pressure gradient $\mathrm{d} \boldsymbol{p}_{0} /\left.\mathrm{d} x_{0}\right|_{i n v}$. For the same $\boldsymbol{A}_{0, i}$, it is also possible to calculate the viscous pressure gradient $\mathrm{d} \boldsymbol{p}_{0} /\left.\mathrm{d} x_{0}\right|_{v}$ by means of the lower-deck equations (3.1) and (3.2). The final set of implicit equations to be solved to find the displacement function $\boldsymbol{A}_{0, i}$ is the difference between the inviscid and the viscous pressure gradients:

$$
\boldsymbol{\Phi}_{0}\left(\boldsymbol{A}_{0, i}\right)=\left.\frac{\mathrm{d} \boldsymbol{p}_{0}}{\mathrm{~d} x_{0}}\right|_{i n v}\left(x_{0, i}\right)-\left.\frac{\mathrm{d} \boldsymbol{p}_{0}}{\mathrm{~d} x_{0}}\right|_{v}\left(x_{0, i}\right)=0, \quad \text { for } i=1, \ldots, N_{x_{0}} .
$$

Equation (3.5) is solved using the Newton-Raphson method by iterating on the displacement function $\boldsymbol{A}_{0, i}$. For additional details, the interested reader can also refer to Sychev et al. (1998).

All the triple-deck calculations were performed using a second-order finitedifference method on an $801 \times 301$ non-uniform mesh with minimum step sizes $\Delta x_{0}=0.005$ and $\Delta y_{0}=0.005$ in the streamwise and wall-normal directions, respectively. Note that, to guarantee independence of the numerical results from the mesh resolution, we performed a mesh refinement study by using a finer grid (one order of magnitude greater resolution than the $\Delta x_{0}$ and $\Delta y_{0}$ provided above). The difference in terms of $L^{\infty}$-norm (normalised with respect to the respective absolute maximum values) between the results on the coarser and finer grids was less than $0.1 \%$ for both the wall shear stress $\tau_{x y}$ and pressure gradient $\mathrm{d} p / \mathrm{d} x$.

The asymptotic dimensionless expansions of the variables (denoted in (3.1) by the subscript ' 0 ') used in the subsonic and transonic regimes are different and they are reported in the following two subsections.

\subsubsection{Subsonic regime}

If we consider the typical triple-deck scales around an isolated roughness element in the subsonic regime (figure $2 a$ ), we can expand the streamwise and wall-normal directions as

$$
\begin{gathered}
x=L\left(1+R e^{-3 / 8} \lambda^{-5 / 4} \mu_{0}^{-1 / 4} \rho_{0}^{-1 / 2} \alpha^{-3 / 4} x_{0}\right), \\
y=L \operatorname{Re}^{-5 / 8} \lambda^{-3 / 4} \mu_{0}^{1 / 4} \rho_{0}^{-1 / 2} \alpha^{-1 / 4}\left(y_{0}+F\left(x_{0}\right)\right),
\end{gathered}
$$

where $x_{0}$ and $y_{0}$ indicate the dimensionless quantities used as asymptotic expansions in the triple-deck approach. Note that we have applied the Prandtl transposition theorem for the vertical coordinate and we have assumed that the hump was fully contained within the lower deck. Concerning the dimensional velocities $(u, v)$, we used the following asymptotic expansions: 


$$
\begin{gathered}
u=u_{\infty} R e^{-1 / 8} \lambda^{1 / 4} \mu_{0}^{1 / 4} \rho_{0}^{-1 / 2} \alpha^{-1 / 4} u_{0}+\cdots, \\
v=u_{\infty} R e^{-3 / 8} \lambda^{3 / 4} \mu_{0}^{3 / 4} \rho_{0}^{-1 / 2} \alpha^{1 / 4}\left(v_{0}+u_{0} F^{\prime}\left(x_{0}\right)\right)+\cdots,
\end{gathered}
$$

where $u_{0}$ and $v_{0}$ are the velocities related to the triple-deck approach. Finally, the dimensional thermodynamic quantities were expanded as follows:

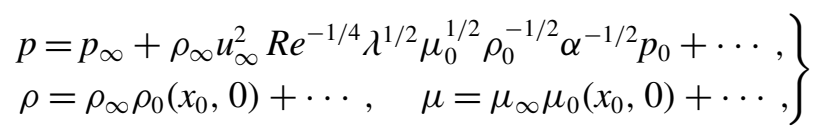

where $\alpha=\sqrt{1-M a_{\infty}^{2}}, p$ is the pressure, $\rho$ is the density and $\mu$ is the dynamic viscosity. Note that these variable transformations were used to compare DNS and triple-deck data in the subsonic case.

\subsubsection{Transonic regime}

In the transonic regime (figure $2 b$ ), the scales for the streamwise and wall-normal coordinates become

$$
\begin{gathered}
x=L\left(1+R e^{-1 / 3} \lambda^{-4 / 3} \mu_{0}^{-1 / 3} \rho_{0}^{-1 / 3} x_{0}\right), \\
y=L R e^{-11 / 18} \lambda^{-7 / 9} \mu_{0}^{2 / 9} \rho_{0}^{-4 / 9}\left(y_{0}+F\left(x_{0}\right)\right),
\end{gathered}
$$

where, again, we have assumed that the hump was fully contained within the lower deck. Concerning the dimensional velocities $(u, v)$, we used the following asymptotic expansions:

$$
\begin{gathered}
u=u_{\infty} R e^{-1 / 9} \lambda^{2 / 9} \mu_{0}^{2 / 9} \rho_{0}^{-4 / 9} u_{0}+\cdots, \\
v=u_{\infty} R e^{-7 / 18} \lambda^{7 / 9} \mu_{0}^{7 / 9} \rho_{0}^{-5 / 9}\left(v_{0}+u_{0} F^{\prime}\left(x_{0}\right)\right)+\cdots .
\end{gathered}
$$

Finally, the dimensional thermodynamic quantities assumed the same expressions as in the subsonic case, with the exception of the pressure, which was expanded as follows:

$$
p=p_{\infty}+\rho_{\infty} u_{\infty}^{2} \operatorname{Re}^{-2 / 9}\left(\lambda^{4 / 9} \mu_{0}^{4 / 9} \rho_{0}^{1 / 9}\right) p_{0}+\cdots .
$$

The Kármán-Guderley parameter was instead

$$
m=\left(1-M a_{\infty}^{2}\right) \lambda_{0}^{-2 / 9} \mu_{0}^{-2 / 9} \rho_{0}^{4 / 9} R e^{1 / 9} .
$$

Note that these variable transformations were used to compare DNS and triple-deck data in the transonic case.

\subsection{Direct numerical simulation}

The governing equations considered for the DNS are the unsteady two-dimensional compressible Navier-Stokes equations.

The spatial numerical discretisation adopted was a spectral $/ h p$ element approach complemented by an explicit (in time) DG method. The temporal discretisation was an explicit fourth-order Runge-Kutta scheme. The spatial discretisation has an order of accuracy equal to $\mathscr{P}+1$, where $\mathscr{P}$ is the order of the polynomials used within each element of the numerical grid. 


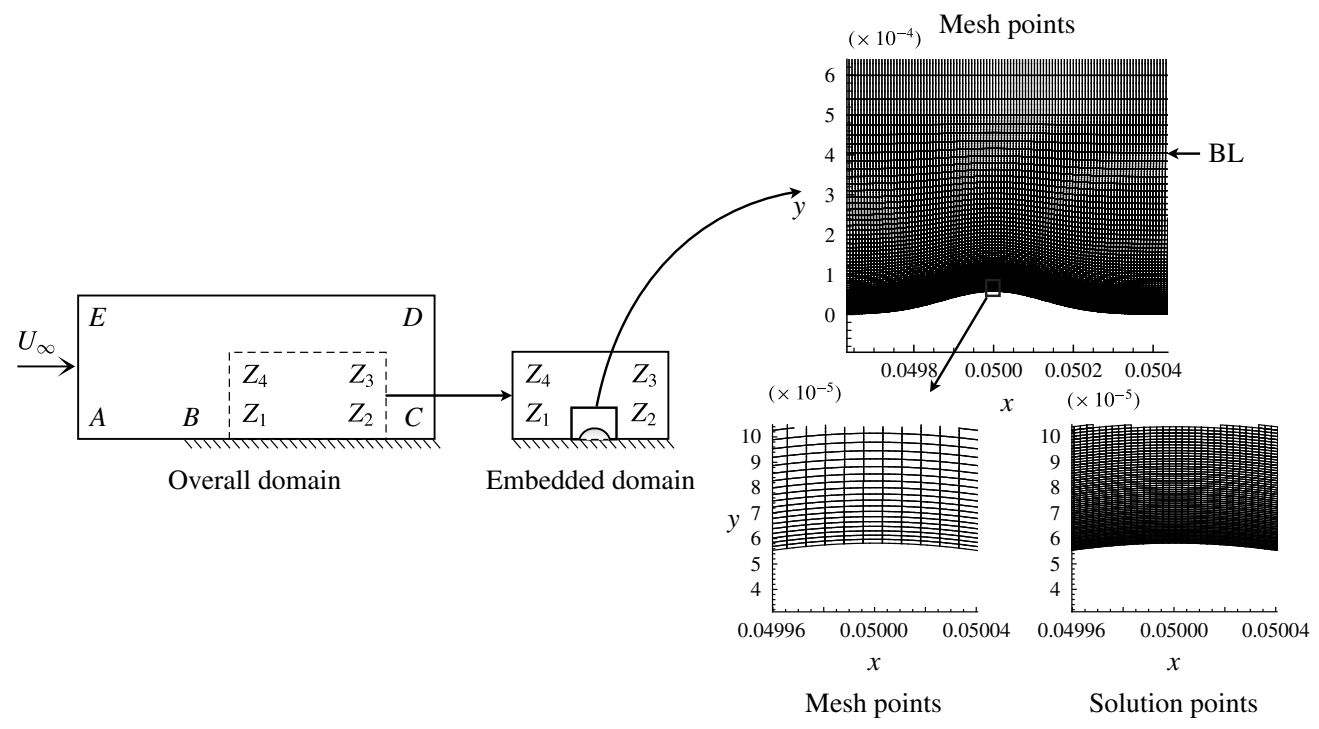

FIGURE 3. DNS configuration.

The numerical setting used is depicted in figure 3. We first simulated a flat plate with no roughness element as represented in figure 3, where we applied symmetry boundary conditions prior to the flat plate $A B$, with $B$ representing the leading edge of the flat plate, which extends up to $C, C D$ is the outflow region, $D E$ is the far field and finally $E A$ is the inflow. In generating the data on the flat plate, we run a sensitivity analysis regarding the position of the boundaries as well as a mesh refinement study in order to assure that the data produced were effectively independent of these factors. All the simulations were run until the steady state was reached. Note that we also verified that the boundary-layer profiles generated were consistent with those calculated by solving the compressible boundary-layer equations under the same conditions.

After having obtained the data on the flat plate without roughness element, we interpolated these data in the embedded domain $Z_{1} Z_{2} Z_{3} Z_{4}$ around the roughness element represented in figure 3. The initial conditions and all the boundary conditions (except the no-slip boundary condition at the wall) were prescribed from the data obtained in the flat-plate simulations. Note that the boundary conditions were Dirichlet and they were imposed in a weak form. Also, for the embedded simulations, we performed a sensitivity study in order to assess the independence of the results from the boundary location as well as from the mesh resolution. The difference in terms of $L^{\infty}$-norm (normalised with respect to the respective absolute maximum values) between the finest mesh used and a mesh with approximately half of the resolution was less than $1 \%$ for both the wall shear stress $\tau_{x y}$ and the pressure gradient $\mathrm{d} p / \mathrm{d} x$.

The final embedded mesh was constituted by $500 \times 66$ elements, where we employed a stretching technique in both the wall-normal and the streamwise directions. We used a solution polynomial of order three within each element and, therefore, the number of solution points in the wall-normal direction was equal to 264 (220 of which were contained within the boundary layer at the roughness location), while that in the streamwise direction was equal to 2000. The minimum $\Delta y$ in proximity to the wall was equal to $2 \times 10^{-6} \mathrm{~m}$. In the streamwise direction, the maximum 

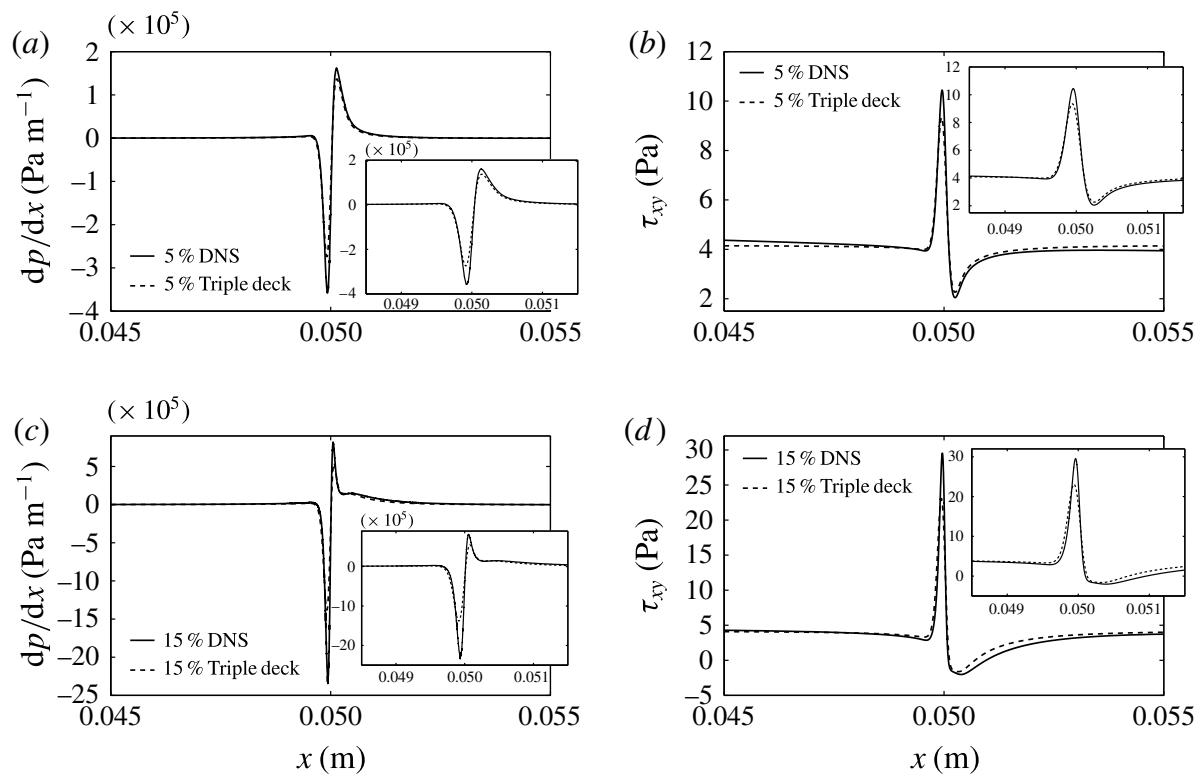

Figure 4. Comparison of $(a, c) \mathrm{d} p / \mathrm{d} x$ and $(b, d) \tau_{x y}$ (dimensional values) for $(a, b)$ the $5 \%$ and $(c, d)$ the $15 \%$ cases, with $M a_{\infty}=0.50$ and $T_{\text {wall }}=T_{\infty}=216.29 \mathrm{~K}$ (case 1 , subsonic).

resolution near the roughness element was equal to $1 \times 10^{-5} \mathrm{~m}$. The description of the roughness element was obtained using a fifth-order spline to achieve an accurate description of the geometry.

\section{Numerical results}

The numerical simulations were performed at two different Mach numbers, the first subsonic, $M a_{\infty}=0.5$, and the second transonic, $M a_{\infty}=0.87$. The shape of the roughness element is given in (2.1), where we used the parameters specified in $\S 2$ and three different heights: $0.05 \delta_{99}, 0.1 \delta_{99}$ and $0.15 \delta_{99}$. We recall that $\delta_{99}$ is a reference value and refers to the Blasius boundary layer at the roughness location.

The comparison between triple-deck and DNS simulations was carried out using two quantities: the pressure gradient along the streamwise direction $\mathrm{d} p / \mathrm{d} x$, and the shear stress $\tau_{x y}$. We also compared the difference between DNS and triple-deck theory in terms of the length of the separation bubbles (where present) as well as their detachment and reattachment points.

In figure 4 we show the comparison of $\mathrm{d} p / \mathrm{d} x$ and $\tau_{x y}$ for case 1: Mach number $M a_{\infty}=0.50$, wall temperature $T_{\text {wall }}=216.29 \mathrm{~K}$ and roughness element heights $h_{r}=$ $0.05 \delta_{99}$ and $h_{r}=0.15 \delta_{99}$, respectively. In figure 5 we show the same quantities for case 2: Mach number $M a_{\infty}=0.87$, wall temperature $T_{\text {wall }}=258.0 \mathrm{~K}$ (see also table 1). From these figures it is possible to see an overall good agreement between triple-deck and DNS data except in proximity to the centre of the roughness element, where large discrepancies are observed (as can be seen from the inset images, which are a closeup view of this region). These discrepancies are expected in the asymptotic analysis because in proximity to the roughness element we observed a larger acceleration of the flow in the DNS results when compared to the triple-deck data. Also, the deceleration of the flow behind the hump is larger in the DNS data when compared to the tripledeck one. These features generate sharper and higher peaks in terms of both wall shear 

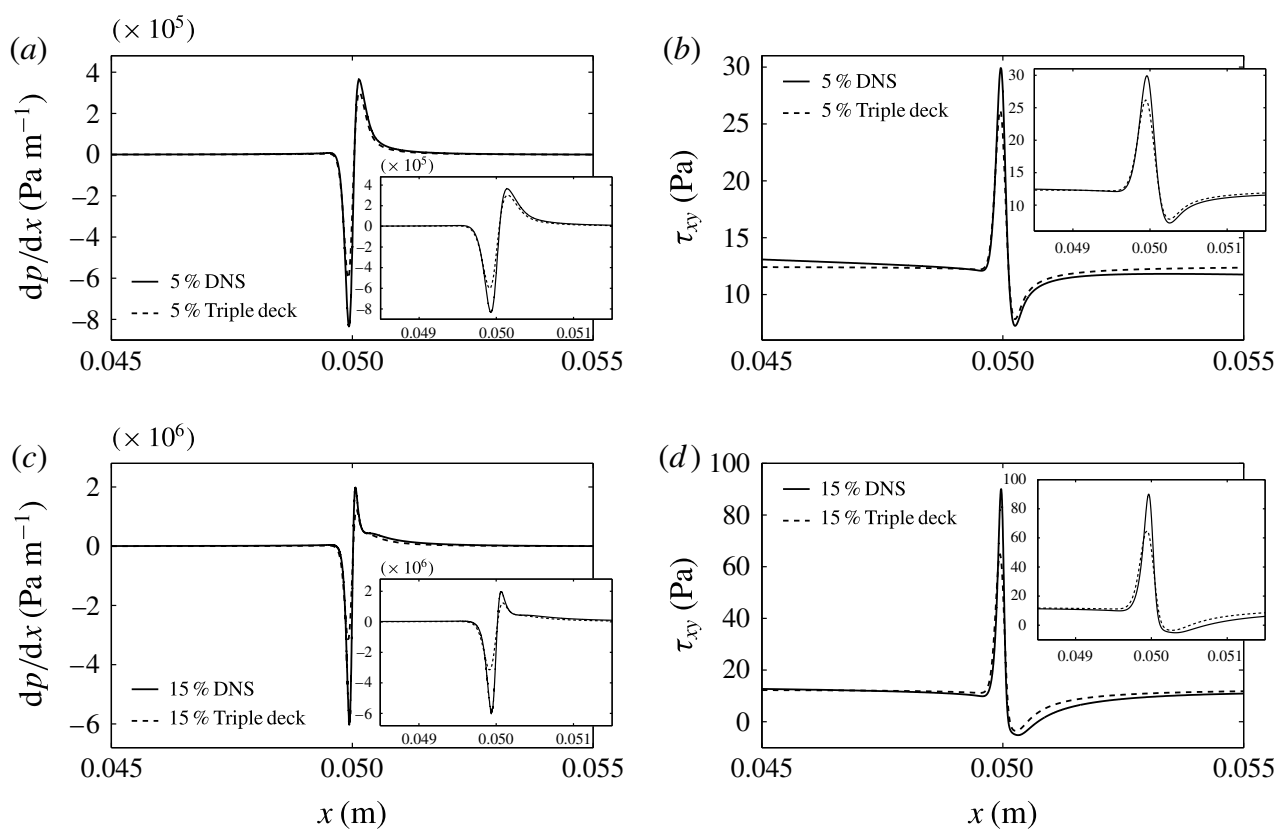

Figure 5. Comparison of $(a, c) \mathrm{d} p / \mathrm{d} x$ and $(b, d) \tau_{x y}$ (dimensional values) for $(a, b)$ the $5 \%$ and $(c, d)$ the $15 \%$ cases, with $M a_{\infty}=0.87$ and $T_{\text {wall }}=258.0 \mathrm{~K}$ (case 2, transonic).

stress $\tau_{x y}$ and longitudinal pressure gradient $\mathrm{d} p / \mathrm{d} x$. We also observed that the wallnormal pressure gradient in the DNS data has a contribution that is neglected in the triple-deck theory and might affect the behaviour of the two quantities investigated and contribute to the discrepancies seen, although some other factors may also play a role.

In table 2 we quantified the differences between DNS and triple-deck data at four distinct locations along the streamwise direction, $\mathrm{P} 1=0.046 \mathrm{~m}, \mathrm{P} 2=0.049 \mathrm{~m}, \mathrm{P} 3=$ $0.051 \mathrm{~m}$ and $\mathrm{P} 4=0.054 \mathrm{~m}$, for both the wall shear stress $\tau_{x y}$ and the longitudinal pressure gradient $\mathrm{d} p / \mathrm{d} x$. We also measured the maximum difference between DNS and triple-deck data (reported in the last column of the table). All the differences are normalised with respect to the maximum absolute value obtained in the DNS results for each case considered. We can see how the difference between DNS and triple-deck data in terms of $\mathrm{d} p / \mathrm{d} x$ and $\tau_{x y}$ in proximity to the hump (points $\mathrm{P} 2, \mathrm{P} 3$ and maximum difference) increases as the height of the roughness element increases in both subsonic and transonic regimes. In particular, we estimated that the maximum difference in terms of $\mathrm{d} p / \mathrm{d} x$ in proximity to the centre of the roughness element was between $27 \%$ (for $h_{r}=0.05 \delta_{99}$, subsonic case) and more than $54 \%$ (for $h_{r}=0.15 \delta_{99}$, transonic case), while moving slightly off from the roughness centre these differences were consistently lower with an order of magnitude below $2 \%$. The maximum differences in terms of $\tau_{x y}$ were between $11 \%$ and $30 \%$ (also in this case normalised with respect to the maximum absolute value of $\tau_{x y}$ obtained from the DNS data) in proximity to the centre of the roughness element. These differences consistently decreased moving either upstream or downstream, with maximum differences in these regions below $4 \%$.

In table 3 we quantified the differences in terms of separation bubble lengths, and the differences in terms of detachment $(D)$ and reattachment $(R)$ points between DNS and triple-deck results. These differences are normalised with respect to the length 


\begin{tabular}{ccccccc}
\hline & $h_{r}$ & $\mathrm{P} 1$ & $\mathrm{P} 2$ & $\mathrm{P} 3$ & $\mathrm{P} 4$ & $\max (\Delta)$ \\
Case $1\left(M a_{\infty}=0.50, T_{\text {wall }}=T_{\infty}\right)$ & $0.05 \delta_{99}$ & $\approx 0.01$ & $\approx 0.16$ & $\approx 0.52$ & $<0.01$ & $\approx 27.54$ \\
& $0.10 \delta_{99}$ & $\approx 0.02$ & $\approx 0.18$ & $\approx 0.87$ & $\approx 0.01$ & $\approx 38.28$ \\
& $0.15 \delta_{99}$ & $\approx 0.03$ & $\approx 0.19$ & $\approx 1.09$ & $\approx 0.05$ & $\approx 44.35$ \\
Case $2\left(M a_{\infty}=0.87, T_{\text {wall }}=258 \mathrm{~K}\right)$ & $0.05 \delta_{99}$ & $\approx 0.03$ & $\approx 0.19$ & $\approx 0.78$ & $\approx 0.06$ & $\approx 29.90$ \\
& $0.10 \delta_{99}$ & $\approx 0.04$ & $\approx 0.20$ & $\approx 1.04$ & $\approx 0.08$ & $\approx 44.34$ \\
& $0.15 \delta_{99}$ & $\approx 0.08$ & $\approx 0.22$ & $\approx 1.08$ & $\approx 0.09$ & $\approx 54.08$ \\
& & & $\left|\Delta \tau_{x y}\right| / \max \left(\left|\tau_{x y D S s}\right|\right)(\%)$ & \\
Case $1\left(M a_{\infty}=0.50, T_{\text {wall }}=T_{\infty}\right)$ & $0.05 \delta_{99}$ & $\approx 1.69$ & $\approx 0.17$ & $\approx 1.12$ & $\approx 1.56$ & $\approx 11.63$ \\
& $0.10 \delta_{99}$ & $\approx 0.87$ & $\approx 0.26$ & $\approx 2.26$ & $\approx 1.03$ & $\approx 20.38$ \\
& $0.15 \delta_{99}$ & $\approx 0.49$ & $\approx 0.60$ & $\approx 3.67$ & $\approx 1.06$ & $\approx 25.56$ \\
Case $2\left(M a_{\infty}=0.87, T_{\text {wall }}=258 \mathrm{~K}\right)$ & $0.05 \delta_{99}$ & $\approx 1.75$ & $\approx 0.17$ & $\approx 1.19$ & $\approx 1.71$ & $\approx 12.47$ \\
& $0.10 \delta_{99}$ & $\approx 0.86$ & $\approx 0.28$ & $\approx 2.21$ & $\approx 1.22$ & $\approx 23.90$ \\
& $0.15 \delta_{99}$ & $\approx 0.28$ & $\approx 0.83$ & $\approx 3.26$ & $\approx 1.20$ & $\approx 30.34$
\end{tabular}

TABLE 2. Difference between DNS and triple-deck theory in the $\mathrm{d} p / \mathrm{d} x$ and $\tau_{x y}$ variables normalised with respect to their maximum absolute values obtained from the DNS data at four different locations along the flat plate: $\mathrm{P} 1=0.046 \mathrm{~m}, \mathrm{P} 2=0.049 \mathrm{~m}, \mathrm{P} 3=0.051 \mathrm{~m}$, $\mathrm{P} 4=0.054 \mathrm{~m}$. The last value is the maximum difference between DNS and triple-deck data, also normalised with respect to the DNS maximum absolute value.

\begin{tabular}{|c|c|c|c|c|c|c|}
\hline & \multirow[b]{2}{*}{$h_{r}$} & \multicolumn{2}{|c|}{ Separation } & \multicolumn{3}{|c|}{$|\Delta(D, R, \mathscr{L})|(\%)$} \\
\hline & & DNS & Triple-deck & $D$ & $R$ & $\mathscr{L}$ \\
\hline Case $1\left(M a_{\infty}=0.50, T_{\text {wall }}=T_{\infty}\right)$ & $\begin{array}{l}0.05 \delta_{99} \\
0.10 \delta_{99} \\
0.15 \delta_{99}\end{array}$ & $\begin{array}{l}\times \\
\checkmark \\
\checkmark\end{array}$ & $\begin{array}{l}\times \\
\checkmark \\
\checkmark\end{array}$ & $\begin{array}{l}-\overline{5.94} \\
2.50\end{array}$ & $\begin{array}{r}\overline{9.79} \\
26.35\end{array}$ & $\begin{array}{l}- \\
15.73 \\
28.85\end{array}$ \\
\hline Case $2\left(M a_{\infty}=0.87, T_{\text {wall }}=258 \mathrm{~K}\right)$ & $\begin{array}{l}0.05 \delta_{99} \\
0.10 \delta_{99} \\
0.15 \delta_{99}\end{array}$ & $\begin{array}{l}\times \\
\times \\
\checkmark\end{array}$ & $\begin{array}{l}\times \\
\times \\
\checkmark\end{array}$ & $\overline{-}$ & $\overline{-}$ & $\frac{-}{26.35}$ \\
\hline
\end{tabular}

TABLE 3. Difference between DNS and triple-deck theory in terms of the detachment point $(D)$, reattachment point $(R)$ and length $(\mathscr{L})$ of the separation bubbles - normalised quantities.

$\ell_{999}$ of the hump (we considered a tolerance $\mathscr{T}=0.001 h_{r}$ at which we measured the length of the hump; this gave us $\ell_{999} \approx 0.00096 \mathrm{~m}$ ).

It is possible to note that the underlying qualitative physics of the problem is well captured by both approaches. In particular, separation happens for the same test cases in both DNS and triple-deck theory. The differences in terms of detachment point is relatively contained (less than $6 \%$ for $h_{r}=0.10 \delta_{99}$ in the subsonic case, and less than $3 \%$ for $h_{r}=0.15 \delta_{99}$ in the subsonic and transonic cases). The major gap between the two approaches is related to the reattachment point, which in turn influences the length of the separation bubble. We note that this gap increases as the height of the hump increases. This is probably related to the local behaviour of the pressure in proximity and prior to the reattachment point. In fact, in the DNS results we observe a larger streamwise pressure gradient in proximity to and behind the hump when compared to the triple-deck theory. Also, the wall-normal pressure gradient is not negligible in this 
region. Both the streamwise and wall-normal pressure gradients strongly influence the deceleration of the flow and therefore the position of the reattachment point; see, for example, Stewartson (1970) and Korolev, Gajjar \& Ruban (2002).

Although the gap is related to the reattachment point and therefore to the length of the separation bubble, we consider the agreement between the two approaches satisfactory.

\section{Summary and conclusions}

In this paper we compared two substantially different approaches, namely DNS and asymptotic triple-deck theory, for calculating the flow past an isolated roughness element on a flat plate with separation behind the element in some situations. The calculations were performed in a compressible regime, and both subsonic and transonic conditions were taken into account. We considered three different roughness element heights, ranging from a non-separated flow to a laminar separation bubble behind the obstacle. The study has been carried out by comparing two quantities, the pressure gradient $\mathrm{d} p / \mathrm{d} x$ and the shear stress $\tau_{x y}$, as well as by evaluating the location of the detachment and reattachment points and the length of the separation bubble where present.

The results obtained for $\mathrm{d} p / \mathrm{d} x$ and $\tau_{x y}$ show an overall good agreement between DNS and triple-deck data except for their maximum and minimum values. This behaviour can be expected since we observe a non-zero wall-normal pressure gradient in the DNS data when compared to the triple-deck results (where a zero pressure gradient along the wall-normal direction is assumed) and a different flow acceleration/deceleration in proximity to the hump between triple-deck and DNS data. This aspect can well affect the local behaviour of the wall shear stress and of the longitudinal pressure gradient, even though some other nonlinear effects can also contribute to the differences observed. In addition, triple-deck theory relies on the assumption that $R e$ is large; therefore we expect a better agreement if we increase $R e$, although the Reynolds number adopted in this work is relevant for aeronautical applications. It is also possible to note that the discrepancy between DNS and triple-deck data increases as the height of the roughness element increases, and therefore when nonlinear effects become more relevant (see table 2 for example).

Concerning the separation bubbles, both DNS and triple-deck theory predict separation for the same test cases. This indicates that the underlying physics of the problem is well captured in both approaches (in the limit of two-dimensional separation bubbles). Specifically, the detachment point compares well, though some rather large differences are present for the reattachment point, probably due to the different longitudinal and wall-normal pressure distributions between DNS and triple-deck data.

This study demonstrates that the asymptotic triple-deck theory captures correctly the qualitative physics and the main flow features in practical aeronautical applications, and therefore it can be effectively used as a reduced model in this field. This is of particular use in estimates of receptivity and in separation prediction studies, where the quantities we have investigated, $\mathrm{d} p / \mathrm{d} x$ and $\tau_{x y}$, are very relevant; see, for example, Rizzetta, Burggraf \& Jenson (1978) and Ruban, Bernots \& Pryce (2013). (Note that the velocity profiles are also important for stability and receptivity calculations. However, the two quantities investigated in this paper, i.e. $\mathrm{d} p / \mathrm{d} x$ and $\tau_{x y}$, provide a satisfactory insight into the separation characteristics of the flow and they can be used for receptivity studies.) 
On the other hand, from a quantitative perspective, the triple-deck theory is not entirely reliable when considering a nonlinear regime and its use in this context has to be considered carefully, especially if accurate quantitative calculations are required.

This work also constitutes the rationale for further studies on the connections between the two approaches, historically developed in two different research communities, and it allows a more theoretical insight into the DNS data as well as a more applied viewpoint for the triple-deck community.

\section{Acknowledgements}

We thank Dr T. Bernots for helpful discussions and Dr H. Xu for his useful comments on the DNS calculations. This work was supported by the Laminar Flow Control Centre funded by Airbus/EADS and EPSRC under grant EP/I037946.

\section{REFERENCES}

Bodonyi, R. J. 1979 Transonic laminar boundary-layer flow near convex corners. Q. J. Mech. Appl. Maths 32 (1), 63-71.

Bodonyi, R. J. \& KLUWick, A. 1977 Freely interacting transonic boundary layers. Phys. Fluids 20 (9), 1432-1437.

Bodonyi, R. J. \& Kluwick, A. 1982 Supercritical transonic trailing-edge flow. Q. J. Mech. Appl. Maths 35 (2), 265-277.

Bodonyi, R. J. \& Kluwick, A. 1998 Transonic trailing-edge flow. Q. J. Mech. Appl. Maths 51 (2), 297-310.

Bowles, R. I. \& SMith, F. T. 1993 On boundary-layer transition in transonic flow. J. Engng Maths 27, 309-342.

Chapman, D. R., Kuehn, D. M. \& LARson, H. K. 1958. Investigation of separated flows in supersonic and subsonic streams with emphasis on the effect of transition. NACA Tech. Rep. 1356.

Cole, J. D. \& CooK, L. P. 1986 Transonic Aerodynamics. Elsevier.

De Grazia, D., Mengaldo, G., Moxey, D., Vincent, P. E. \& Sherwin, S. J. 2014 Connections between the discontinuous Galerkin method and high-order flux reconstruction schemes. Intl $J$. Numer. Meth. Fluids 75, 860-877.

Hesthaven, J. S. \& Warburton, T. 2008 Nodal Discontinuous Galerkin Methods - Algorithms, Analysis and Applications. Springer.

Hsiao, C.-T. \& Pauley, L. L. 1994 Comparison of the triple-deck theory, interactive boundary layer method, and Navier-Stokes computation for marginal separation. Trans. ASME: J. Fluids Engng 116 (1), 22-28.

Karniadakis, G. E. \& Sherwin, S. J. 2005 Spectral/hp Element Methods for Computational Fluid Dynamics. Oxford University Press.

Korolev, G. L., GajJar, J. S. B. \& Ruban, A. I. 2002 Once again on the supersonic flow separation near a corner. J. Fluid Mech. 463, 173-199.

Kravtsova, M. A., Zametaev, V. B. \& Ruban, A. I. 2005 An effective numerical method for solving viscous-inviscid interaction problems. Phil. Trans. R. Soc. Lond. A 363 (1830), $1157-1167$.

Lighthill, J. 2000 Upstream influence in boundary layers 45 years ago. Phil. Trans. R. Soc. Lond. A 358 (1777), 3047-3061.

Martin, M. P. 2007 Direct numerical simulation of hypersonic turbulent boundary layers. Part 1. Initialization and comparison with experiments. J. Fluid Mech. 570, 347-364.

Messiter, A. F. 1970 Boundary-layer flow near the trailing edge of a flat plate. SIAM J. Appl. Maths 18, 241-257.

Moin, P. \& MAHESh, K. 1998 Direct numerical simulation: a tool in turbulence research. Annu. Rev. Fluid Mech. 30 (1), 539-578. 
NeILANd, V. Y. 1969 Theory of laminar boundary-layer separation in supersonic flow. Mekh. Zhidk. Gaza 4, 53-57.

Pirozzoli, S., Grasso, F. \& GATSKi, T. B. 2004 Direct numerical simulation and analysis of a spatially evolving supersonic turbulent boundary layer at $m=2.25$. Phys. Fluids 16, 530-545.

Rizzetta, D. P., Burggraf, O. R. \& Jenson, R. 1978 Triple-deck solutions for viscous supersonic and hypersonic flow past corners. J. Fluid Mech. 89 (3), 535-552.

Rizzetta, D. P. \& Visbal, M. R. 2014 Numerical simulation of excrescence generated transition. AIAA J. 52 (2), 385-397.

Rizzetta, D. P., Visbal, M. R., Reed, H. L. \& Saric, W. S. 2010 Direct numerical simulation of discrete roughness on a swept-wing leading edge. AIAA J. 48 (11), 2660-2673.

Ruban, A. I., Bernots, T. \& PRYCE, D. 2013 Receptivity of the boundary layer to vibrations of the wing surface. J. Fluid Mech. 723, 480-528.

Stewartson, K. 1969 On the flow near the trailing edge of a flat plate II. Mathematika 16, $106-121$.

Stewartson, K. 1970 On laminar boundary layers near corners. Q. J. Mech. Appl. Maths 23 (2), $137-152$.

Stewartson, K. \& Williams, P. G. 1969 Self-induced separation. Proc. R. Soc. Lond. A 312, 181-206.

SyCHEV, V. V. 1972 Laminar separation. Fluid Dyn. 7 (3), 407-417.

Sychev, V. V., Ruban, A. I., Sychev, V. V. \& Korolev, G. L. 1998 Asymptotic Theory of Separated Flows. Cambridge University Press.

Timoshin, S. N. 1990 Asymptotic form of the lower branch of the neutral curve in transonic boundary layer. Uch. Zap. TsAGI 21 (6), 50-57. 